\title{
Visualizing the (in)Visible: Mapping Culture and the Forces that Shape the Urban Experience
}

\author{
Awilda Rodriguez \\ Oklahoma State University, USA \\ awilda@okstate.edu \\ Paolo Sanza \\ Oklahoma State University, USA \\ Paolo.sanza@okstate.edu
}

\begin{abstract}
Digital technologies not only have made information accessible but also have exponentially generated new data. The continue growth of urban GIS and pervasive computing is helping us analyze human interactions in unprecedented ways. Yet, when researching urban sites, the students' task is conventionally limited to the analysis of aggregate spatial data that does not reveal the invisible forces acting upon the site. The limitation of this approach is further evident if students cannot visit the site. This paper explores the role of information visualization to uncover uncommon site forces and to supplement students' lack of embodiment with far distanced sites.
\end{abstract}

Keywords: Information visualization; Mapping, Urban environments.

Architecture has not only always been concerned with graphic representation, but it has been its principal language. Today, the advancement of digital technologies is significantly changing our knowledge of the world as well as exponentially generating new data. Urban environments research within schools of architecture has been traditionally limited to the analysis of aggregate spatial data. The shortcoming of such analysis lays on the fact that it prevents to expose the temporal and micro-scale elements that make up the entire multi-dimensionality of an urban experience.

Architects, landscape architects, urban designers as well as graphic designers find themselves working mostly in and reacting to existing urban environments made of "multiple layers of urban morphologies, physical conditions and narratives". (Kühl 2009) Often, our understanding of place is superficial, and manipulated by our perceptions. This holds more truth when we are invited to work on urban environments far from our place of working residence. How then our pre-existing knowledge and perception evolves and matures through the process of collecting information about an urban site? Addressing the task of "collecting data" has recently changed as a result of developments in information technologies. Consequently, we can now apply unconventional methodologies to viewing, analyzing, understanding, and eventually managing the constant flux of information that characterize [complex] urban environments. New information visualization techniques, for instance, are today more than innovative statistical and computational algorithms visualizations. (Abrams 2006)

We can speculate that it is possible to read a site differently if were to alter our means of sight. For instance, if we were to record a site through a webcam, the results, and our own reading and understanding of the site itself, would be different from, for instance, the recording of the site via an infrared device, or a device recording the heat emanating from any animated or unanimated body. Naturally, if we were to close both of our eyes, our reading of the site would provide us of knowledge that may have not been fully appreciated or registered with our eyes open, such as sounds, smells, or the tactile texture of a surface when caressed by our bare hands or feet. Regrettably, students may not always have the opportunity to walk the site. It is then paramount to provide them with means that can supplement their lack of embodiment.

The task of data collection and mapping are of primary significance to unveil site properties. If the temporal site forces are identified, the architecture can be developed to change with these forces, creating an intelligent design response. Maps, aerial photographs, zoning surveys, census charts, and other spatial data are "instrumental materials to engage with when looking down upon a subject site to obtain a general overview" (Kühl 2009), yet they portray a level of abstraction, and do not necessary render visible the sense of place. How then we can find a process that can facilitate uncovering the daily realities of a site? For starters, we need a critical reassessment of the conventional approaches to site analysis, program, infrastructure, and form.

A studio exercise given to a recent 5th year architectural design studio sought to exploit the pedagogic potential of research as a form of design based critical inquiry. What are the contemporary forces that act in a multi-scalar level? And which are the disciplines that negotiate between the interdisciplinary characters that dynamically shape a space? In the exercise, while traditional 
concerns of site analysis where not neglected, students were invited to unveil other site facets and truths and engage in the realms of disciplinary knowledge associated with other complementary site issues such as ecology, spatial anthropology, art, economy, politics, etc. not solely found within or around the physical sizes of the site(s), but rather embracing a much assigned larger territory which, we believed, was paramount to help disclosing intrinsic characteristic of the site(s) which were located with the metropolitan area of San Juan, Puerto Rico, 1,700 miles away from the design studio

When working on foreign regions, the traditional methods of in situ research tend to emphasize the visual and external appearance; the focus is on the "street scene" or façade analysis. This type of investigation, however, does not uncover those personalities of the urban environment that, for instance, are directly correlated with its current users. Are there any systematic frameworks that can uncover these elusive or invisible forces that so profoundly define the quality and shape of a specific environment?

The task was accomplished by using information visualizations techniques in order to facilitate students' comprehension of the multi-dimensional layers and invisible forces that act upon a site and that ultimately and uniquely shape it. Information visualization tools permitted students to observe, analyze and manipulate complex information peculiar to the natural and cultural environment of the site(s), and revealed a much more comprehensive understanding of the site. The integration of visualization tools into an urban research can be compared to terrain mapping. (Dorling 1997) Through a broader, yet focused analysis, students were able to discover underlying patterns of information that could drive knowledge-based and novel solutions for the "in progress" reality of the design problem, while strongly expressing the specific of the culture and locale.

At first, students found hard expressing graphically those temporal and ephemeral actions acting upon the site(s), which, albeit invisible, have a strong physical expression. Yet by posing them questions such as "What is the character of the space in relation to the forces that create a sense of place or genius loci," or "What graphic languages are appropriate to capture the atmosphere of a place in a qualitative measure? Or even the lack of place?" helped them distance themselves from representing the exercise by reevaluating past analyses experiences and instilled in them an augmented sense of curiosity and challenge. As the exercise did not ask individual students to uncovered everything about each of the two territories assigned, but to respond to one sole personal quest, then to be shared among the class and inserted in "collective" knowledge about the site(s), students were able to identify and recognize that they could experience a certain indefinable well being about the site(s) that needed to be visited over and over to be able to be expressed and (well) communicated.

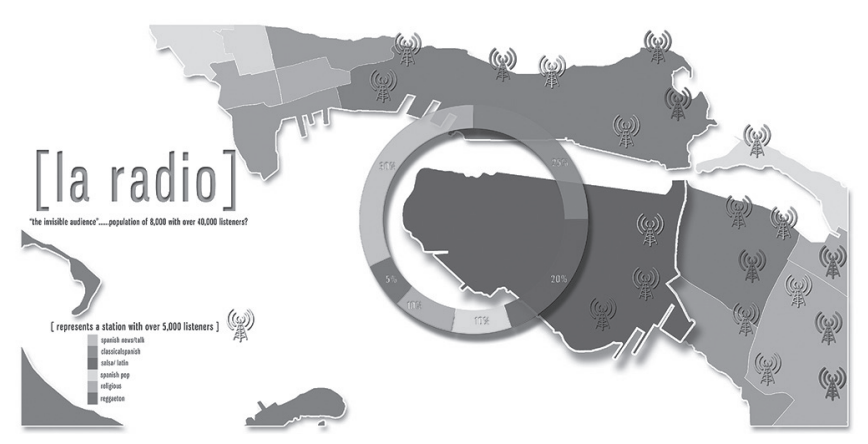

Figure 1: Mapping the invisible: Radio stations listeners as an alternative mean to identify the demographics surrounding site $1 \bullet$ Sean Ryan Frans

Because of the constant flux of information circulating thru the world wide web at any point, students found information that was fresh and relevant as well as obsolete and superficial, but more importantly they recognized that this state of rapid change had a direct correlation with the growth or contraction of any particular social aspects of the urban environment in question. It subsequently helped them solidifying the abstract notion of temporal dimensions by identifying distinct customs. Students, for instance, recognized in elements such as food street vendors, food networks, festivals and open-air musical performances, crime patterns, real estate value, and even airlines' route schedules, social patterns that had a great impact on the sense of place. Others discovered that the cruise ships embarking and disembarking hours completely changed the feel and character of Old San Juan. At the visible scale, the size of the cruise ships dwarfed the colonial town; yet, the impact of rapid and temporary change of population due to the simultaneous disembarking of thousands of passengers quickly modified the urban choreography of the city.
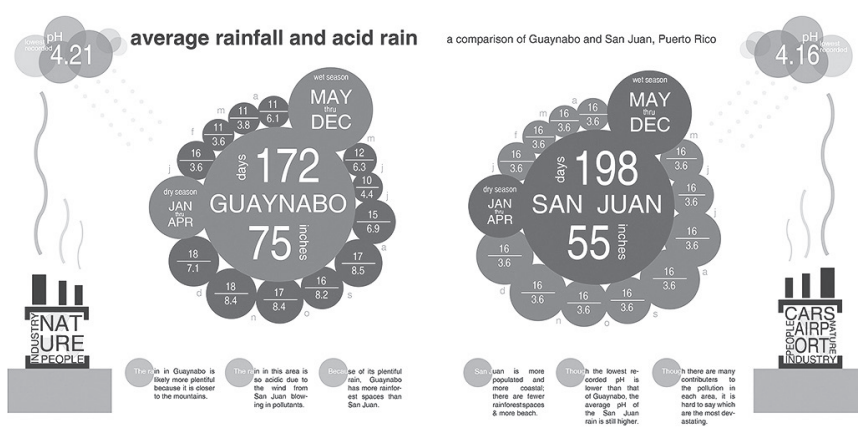

Figure 2: Mapping precipitation and acid rain in both sites $\bullet$ Danna Colbert

The methodology for visualizing the data was established following the students' individual investigation of various local urban forces, dissected to extrapolate and isolated different level of complexity, and then communicated by operating in-between artistic and scientific grounds. The method employed, therefore, followed a sequential protocol from initial analysis to a proposed hypothesis and model of representation to a speculative pattern of construction to the final original (graphic) visualization of the information. (Katz 2012) Tools employed to support each 


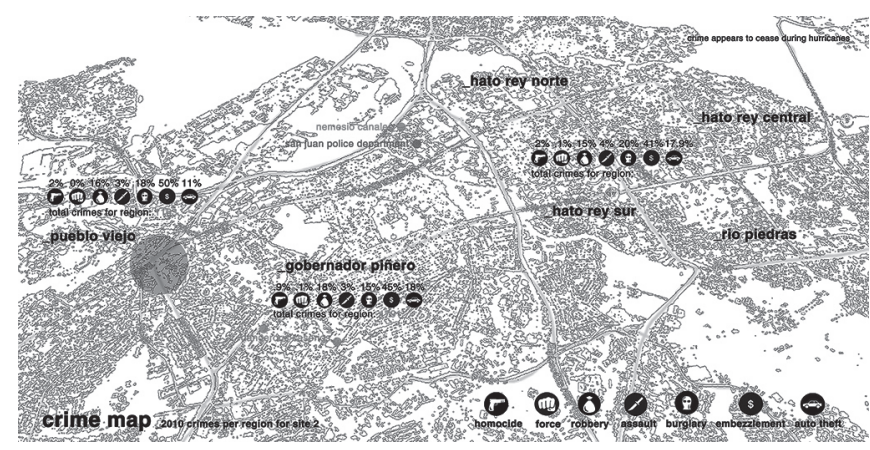

Figure 3: Mapping crime around site 1• Tanner Hall

student's inquisitiveness included social networks, aerial surveys and virtual navigations, web-based image gathering conducted via Google Earth or Virtual Earth, GIS, and live-streaming information such as traffic patterns, local blogospheres, crowd sourcing software, gapminder.org, etc.

Why such a strong emphasis on the visual and the graphic method? It responded to our belief that how an urban site is represented determines students' site knowledge, and can augment the intimate relationship that students ought to have with it in order to create architectural propositions truly grounded in their settings. The exercise created an opportunity within the design studio to explore methods not only to communicate clearly and effectively atypical site forces, but also to sharpen the student critical skills needed to test and refine their imminent urban design strategies in respond to an organism, such is the city, in continuous flux.
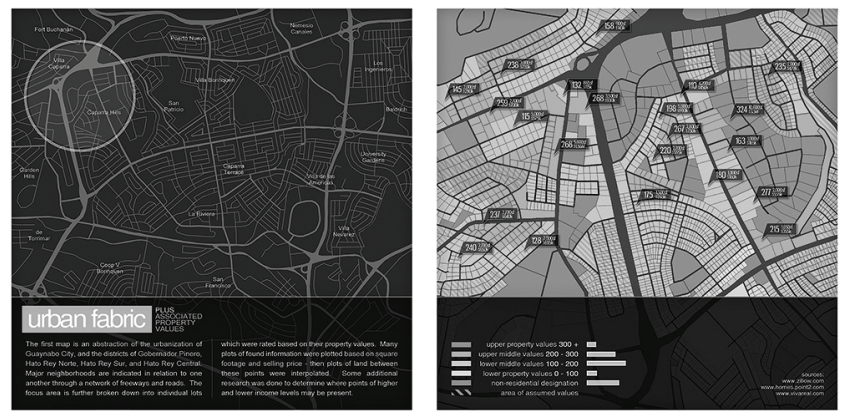

Figure 4: Mapping real estate value around site $2 \bullet$ Jessica Shelton

In order to properly represent graphically the various flows of interaction investigated or discovered, students needed to acquire the proper core skills for visual analysis in order to decompose the complexities involved on urban dynamics. It was not only about assembling a pool of information, but rather identify the logical process to break down the elements or components in a way to facilitate organization and analysis to eventually develop a visualization strategy in order to make it "knowledge" (Lima 2011). The process of synthesize the information gathered and breaking it down to smaller components in order to comprehend it as a whole, helped students understanding how the multiple sub-data each student investigated interconnected. Thus the process of sorting uncovered the relationships between the parts.
We introduced students to the grammar of graphics: data, variables, scales, dimension, sorting, geometry, aesthetics, and statistics. (Wilkinson 1999) During the data analysis students' appropriated the research methods commonly used by statisticians and computer scientist, which also utilize data visualizations. Data is then the main ingredient for information visualization graphics. Dimensioning was a good tool for students to start connecting different data sets that influenced each other. Combining several scales within one graphic output allowed them to see the cause and effect of data. (Few 2009) The visualization that quantified crimes and arrest within the site and immediate surroundings, for instance, exemplifies such strategy. By adding a further dimension which portrayed gender committed crimes, or emphasized that certain type of wrongdoing, such as embezzlements, were concentrated in certain professions or neighborhoods, the student was able to portray social patterns, and further fuel the class understanding of the place.

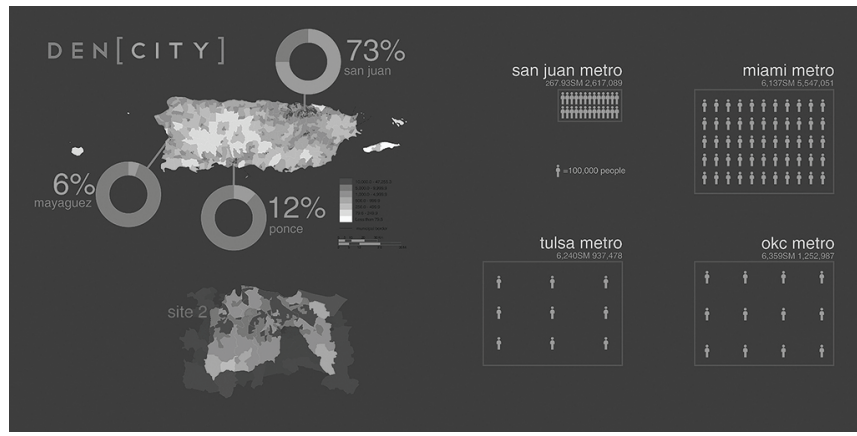

Figure 5: Mapping population density and comparing it with familiar urban environments • Jonathon Pontious

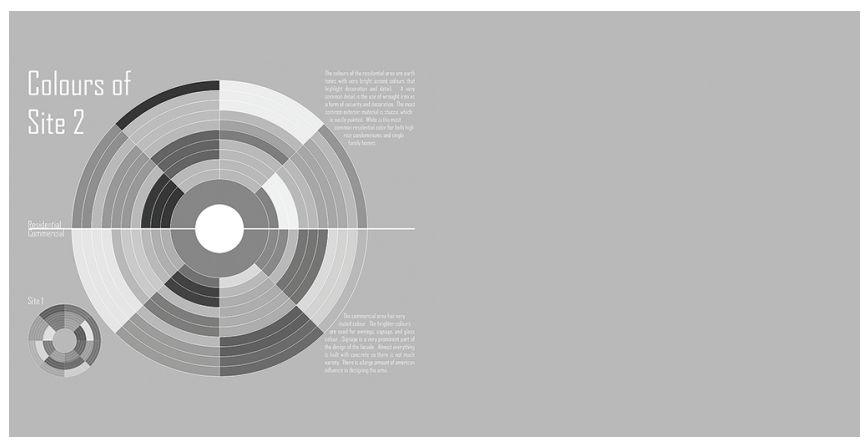

Figure 6: Mapping the colors of buildings surrounding site 2 and compare them with those found on site $1 \bullet$ Cristin Butler

All throughout the process of sorting data we witnessed in students, as expected, diverse cultural biases, personal preferences, priorities and preconceptions. Yet, students sought a conceptual reconstruction and fusion of the physical and digital site(s) that transcended their physical limitations. Recognizing that they were still outsider "looking in", they embraced the exercise as an opportunity to contribute and exchange knowledge, and offering reinterpretation, or even alternate meanings, to the discovered local forces.

The pedagogic effectiveness of any new methodology always needs to be carefully considered and planned to anticipate quality 
results. One detail that we did not consider was the language barrier. Students found a great deal of information that needed to be translated, but the state of current web translators software is not yet able to translate correctly local mannerisms or expressions, or a meaningful and logical language syntax for that matter. Important data was then lost in translation. Nevertheless, the visualization methodology implemented for the site analysis resulted on not only making possible that students understood the complexity and local essence of the foreign urban environments and recognized the multi-dimensionality of their challenges, but also allowed them to identify connections and apprehend the social-economic dynamics of the place(s). By then comparing the researched information with similar actions found or not found in their own local environment allowed them to recognize the relationship between physical space, established networks and local actions.

\section{References}

Abrams, J, \& P. Hall. (2006). Else/where : mapping. Minneapolis, Minn, University of Minnesota Design.

Dorling, D, \& D. Fairbairn. (1997). Mapping : ways of representing. Harlow, Essex, England, Longman.

Few, S. (2009). Now you see it : simple Visualization Techniques for Quantitative Analysis.. Oakland, Calif, Analytics Press.

Katz, J. Designing information : human. , 2012. Print.

Kühl, K.. (2009, Month. Day ). In URBAN DESIGN STUDIO I: THE 5 BOROUGH STUDIO. Retrieved Jul. 10, 2013, from http://www.msaudcolumbia.org/summer/?page_id=37

Lima, M. (2011). Visual complexity : mapping pa. New York, Princeton Architectural Press.

Wilkinson, L, \& G. Wills. (1999). The grammar of graphics. New York, Springer. 